\title{
Epithelial ovarian cancer: A feasible plan for adjunctive treatment using simultaneous acyclovir, ambrisentan, captopril, disulfiram, fluvoxamine- augmented ramelteon, icatibant, imiquimod peritoneal lavage, and plerixafor
}

\author{
R.E. Kast ${ }^{*}$, \\ Department of Psychiatry, University of Vermont, 22 Church Street, Burlington, VT 05401, USA. \\ richarderickast@gmail.com
}

\begin{abstract}
Background: To improve current treatment options in epithelial ovarian cancer, this paper outlines how nine non-cytotoxic drugs, eight of which are marketed for non-cancer indications, can be expected to augment current cytotoxic chemotherapy. By matching what we know about the pathophysiology of epithelial ovarian cancer with the pharmacologic attributes of currently marketed non-cytotoxic drugs, nine drugs were found to match eight identified important growth facilitating paths. The nine drugs: 1) The old anti-viral drug acyclovir inhibits indolamine 2, 3- dioxygenase, an action expected to lower immunoinhibitory Treg lymphocytes; 2) Ambrisentan, marketed to lower pulmonary hypertension can be expected to lower endothelin-1 mediated growth stimulation; 3) The old anti-hypertension drug captopril inhibits matrix metalloproteinases-2 and -9 . Both are used for invasion and growth signaling; 4) The old drug disulfiram used to treat alcoholism can be expected to inhibit stemness; 5 and 6) Ramelteon, a melatonin agonist marketed as a sleep aid also stimulates interleukin-2 synthesis. The antidepressant fluvoxamine increases ramelteon levels thereby amplifying interleukin-2 stimulating effects; 7) Imiquimod is a topical Toll-like receptor-7 agonist marketed to treat basal cell carcinoma and other skin cancers by host immunostimulation. It cannot be given systemically but is tolerated well intraperitoneally and can be expected to behave in a similar way in ovarian cancer, causing regression of topically exposed micrometastases after imiquimod peritoneal lavage; 8) The CXCR4 chemokine signaling receptor that plays an important role in many cancers including epithelial ovarian cancer, is blinded by plerixafor, a drug approved for bone marrow stem cell mobilization; 9) Bradykinin is active in generating ovarian cancer ascites. Also captopril risks generating supraphysiological bradykinin levels. Both aspects necessitate using icatibant, a bradykinin blocker. Such a large number of drugs opens the way to unexpected side effects but there is no immediately apparent interaction of concern with simultaneous use of these. If effective as predicted, this type of drug mix could be elevated to a principle of Comprehensive Growth Factor Inhibition as a new core treatment path in cancer.
\end{abstract}

Preamble: "Little minds try to defend everything at once, but sensible people look at the main point only; they parry the worst blows and stand a little hurt if thereby they avoid a greater one. If you try to hold everything, you hold nothing." Frederick the Great, mid-1700's.

\section{Introduction}

Five year overall survival for epithelial ovarian cancer, EOC, is about $50 \%$, a somewhat improved number from a decade ago but clearly better treatments are needed and are being actively sought [1-4]. In our effort to find new adjunctive treatment approaches to improve the current prognosis of EOC we searched the published research data for growth factors documented to be active in promoting EOC development and growth for which we happen to have already-marketed and approved drugs that have been shown to inhibit the given growth factor. This short note presents the preliminary results of that endeavor, having identified eight growth enhancing paths for which we have nine already-marketed drugs to block or constructively influence.

If effective we might be able to elevate the concept of Comprehensive Growth Factor Inhibition, CGFI, to the level of a fundamental arm of cancer treatment generally, to be used along side of surgery, current cytotoxic-based drugs and irradiation. This short note gives specific details for a CGFI plan in EOC treatment.

Although unwieldy and somewhat un-esthetic, the simultaneous use of the suggested nine drugs does not pose any specific threat. No particular side effect or amplified toxicity would be predictable or immediately evident from reviewing the individual drugs' pharmacology and side effect profile. This does not exclude unpleasant surprises. With close observation and tight follow up we believe the gravity of EOC warrants taking a cautious risk here- the Preamble above applies.

We can liken our concept of CGFI to the military concept of nonkinetic operations. In the military's words: "Kinetic operations are actions in the battlespace that involve direct fires, indirect fires and other resources specifically intended to violently kill the enemy. Non-kinetic operations are actions in the battlespace that shape the environment without directly engaging the [enemy]

(c) 2012 Kast; licensee Herbert Publications Ltd. This is an open access article distributed under the terms of Creative Commons Attribution License (http://creativecommons.org/licensesby/2.0), This permits unrestricted use, distribution, and reproduction in any medium, provided the original work is properly cited 
with violent weaponry" [5]. CGFI generally, and the nine drugs specifically discussed in this note for EOC, constitute our non-kinetic operations. Surgery, irradiation, and cytotoxic chemotherapies correspond to our kinetic operations.

\section{The Leaky Bucket problem}

With many overlapping growth promoting signaling systems, inhibition of any one or even few of these systems will result in no or little increase in overall survival. This situation can be likened to a bucket with ten holes in the bottom. The effect of plugging one or two holes would not be evident; the remaining open holes would just take a little more flow to compensate. It would only be after most holes are plugged that a longer emptying time [corresponding to longer overall survival] would be noticed.

\section{NINE PROPOSED DRUGS AND THE CORRESPONDING EOC SYSTEMS}

\section{Inhibit EOC endothelin-1 signaling with ambrisentan:}

Expression of both the 21 amino acid peptide signaling molecule endothelin-1 and its receptor ET-A have been extensively documented in EOC cells, $[6,7]$ where expression has been shown to enhance epithelial-tomesenchymal transition and relative resistance to current cytotoxic chemotherapies $[6,7,8]$. Ambrisentan is a 378 Da orally available ET-A inhibitor, approved and marketed for treatment of pulmonary hypertension in both the EU and USA/Canada [9]. By blocking endothelin-1 signaling in the course of chemotherapy for EOC we would deprive EOC cells of this growth and angiogenesis ET-A signaling path, thereby enhancing chemotherapy effectiveness [6].

\section{Reduce MMP-2 and MMP-9 activity with captopril:}

Rodent studies, both in vitro [Matrigel invasion, histochemistry, and similar] and in vivo with EOC cell lines have previously shown a prominent role for two proteases, matrix metalloproteinase-2, MMP-2, also known as $72 \mathrm{kDa}$ gelatinase-A, and MMP-9, also known as $92 \mathrm{kDa}$ gelatinase-B [10-14]. Studies directly looking at MMP-2 and MMP-9 in resected human EOC tissues [15-23] show MMP-2/MMP9 overexpression, with evidence that degree of MMP overexpression is inversely related to survival duration [lower expression, longer survival] in some studies [15,19,21].

MMP-2 and -9 are thought to have roles in degradation of the extracellular matrix allowing EOC migration as well as in triggering growth-promoting surface receptors via proteolytic clipping of surface-tethered ligands for these receptors [24]. MMP-2 and MMP-9 are synthesized as pro-molecules that require proteolytic cleavage themselves before they gain their own proteolytic activity [16,22]. Human EOC biopsy tissue contains the necessary protease to generate active MMP-2 from INACTIVE pro-MMP-2 [16].

Starting with the 1993 in vitro data of Sorbi et al. [25] showing that gelatinase activity of both MMP-2 and MMP-9 were inhibited by captopril, several human studies of this have been carried out, reviewed below. Captopril is an angiotensin converting enzyme, ACE, inhibitor in use since 1979 to treat hypertension and as a renal protective agent [26]. Currently, due to captopril's short half-life requiring multiple daily doses, more recently introduced ACE inhibitors allowing once daily dosing are usually used clinically.

Captopril inhibited in vitro migration and MMP-2 and MMP-9 activity of two glioblastoma cell lines and short term biopsy tissue culture [27]. Nakagawa et al. showed earlier that captopril inhibited both gelatinase and migration in glioma cell line T98G [28]. A single $75 \mathrm{mg}$ oral captopril dose reduced CSF ACE level by $60 \%$ indicating robust captopril blood-brain barrier penetration [29].

Captopril inhibited MMP-2 activity in peritoneal dialysis fluid [30]. Serum MMP-9 increases during acute Kawasaki disease and this MMP-9 activity was inhibited by ex vivo serum exposure to captopril [30]. In vitro plasma from acute myocardial patients showed inhibited ACE and MMP-9 activity [31]. Heart failure patients had increased MMP-9 within the failing myocardium that was inhibited by in vitro exposure to captopril [32]. MMP-2 and MMP-9 activity was reduced to half at captopril concentrations of 30 to $50 \mathrm{nM}$ [25], levels easily clinically achieved in humans.

Although most of the research on MMP's and ACE inhibitors has been done with captopril, other commercially marketed pharmaceutical ACE inhibitors do have evidence of MMP-2 and -9 inhibition. Cardiac biopsy material showed upregulated MMP-2 and or MMP-9 in various heart failure states that was inhibited in vitro by both captopril and the related ACE inhibitor ramiprilate [33]. The recent demonstration by Efsen et al [34] that ramiprilate inhibits Crohn's fistula MMP-9 activity [34] is strong support for the clinical use of captopril or the related ACE inhibitor ramiprilate to decrease EOC's ability to use MMP-2 and MMP-9 to grow and thrive.

\section{Decrease Tregs with acyclovir:}

Tregs are a sub-population of lymphocytes that are thought to inhibit or down-regulate other lymphocytes' immune responses [35]. Tregs bear CD4+, CD25+ FoxP3+ surface markers. Among several immune system inhibitory factors identified in EOC, prominent among these are elevated Tregs in EOC [36-45] with some researchers finding higher tissue Tregs correlate with shorter survival [41-45].

Indolamine 2-3-dioxygenase, IDO, is the enzyme that converts tryptophan to $\mathrm{N}$-formylkynurenine, which is the initial step in tryptophan catabolism. IDO helps generate immunosuppressive Tregs [46-48] leading to obvious pharmaceutical attempts to increase immune responses in cancer treatment by inhibiting IDO [fewer Tregs] and alternatively damping immune responses [more Tregs] by increasing IDO as for example a treatment path in multiple sclerosis or systemic lupus erythematosis $[46,49]$.

The old drug acyclovir, one of the first anti-viral drugs, was shown to inhibit IDO [50]. If the pre-clinical data holds, such IDO inhibition will decrease Tregs, a potentially useful intervention in EOC given the documented Treg upregulation in this disease. Particularly intriguing and important in this 
context are recent reports of increased IDO in EOC [51,52]. This could interestingly enough account for the findings of elevated circulating blood Tregs in EOC compared to controls, and most tellingly the fall in Tregs after surgical debulking of EOC [53].

Others are thinking along similar lines, seeking to reduce Tregs during EOC $[54,55]$. One approach is to use low-dose cyclophosphamide to reduce Tregs prior to and during experimental vaccination in EOC patients in the effort [56]. Another approach is to use a toxin linked to the natural ligand for CD25 [57], when binding would then be suicidal for Tregs and any other CD25 bearing cell. If the preclinical data holds in the actual clinical situation then acyclovir might be a safer, more effective substitute for either of these.

\section{Defeating stemness with disulfiram:}

Disulfiram is a small molecule inhibitor of aldehyde dehydrogenase, ALDH. Ethanol is catabolized to acetaldehyde that in turn is broken down to acetic acid by ALDH [58]. By inhibiting this last step, disulfiram, commonly given as a single $250 \mathrm{mg}$ tablet once daily, results in large amounts of acetaldehyde building up if ethanol is ingested. This in turn gives intense nausea, vomiting, malaise, flushing. In the absence of ethanol ingestion disulfiram usually is un-noticed by the patient and is side effect free. Disulfiram has been in continuous use worldwide to treat alcoholism for over sixty years $[58,59]$.

High ALDH activity is quite commonly associated with other attributes of stemness in both cancer and normal stem cell sub-populations $[60,61]$. Stem cells with a higher expression of ALDH show a higher proliferation rate, higher clonal plating efficiency, and increased resistance to cyclophosphamide and doxorubicin [61]. These authors documented good ALDH inhibition by disulfiram and a corresponding reduction in stemness attributes [61], thereby confirming earlier predictions [59] that disulfiram can defeat stemness via ALDH inhibition.

Human EOC stem cells over-express ALDH and this ALDH over-expressing sub-population shows enhanced proliferation or growth advantage [over the lower or non-ALDH expressing sub-population], better plating efficiency, and greater chemotherapy resistance [62-66].

\section{Interleukin-2 increase with fluvoxamine- enhanced ramelteon:}

Melatonin is a serotonin metabolite that is synthesized by many cells of the body, including lymphocytes [67]. A large research database supports an immune enhancing role for melatonin by unknown mechanisms $[67,68]$ leading to suggestions that melatonin might have a role in immune enhancement in cancer or during cancer vaccine trials $[67,69]$

A melatonin analogue marketed as a sleep aid, ramelteon, stimulates and binds to melatonin receptors. with several times greater affinity than the natural ligand melatonin [70]. Since melatonin receptors are present on a sub-population of lymphocytes and melatonin and agonists at melatonin receptors stimulate interleukin-2, IL-2, synthesis and release
[71-73] this is thought to be a prominent immunostimulatory path of melatonin. Fluvoxamine increases ramelteon levels by a factor of $x 16$ thereby potentiating ramelteon's stimulation of the melatonin receptor [71].

A further benefit could derive from the observation that we have evidence that IL-2 converts Treg to T effector cells and lose their immunosuppressive capacity $[44,74]$. Note that Tregs bear CD25+, the outer cell membrane receptor for IL-2. Thus Treg decreases and increased IL-2 could both be achieved by fluvoxamine-augmented ramelteon. "Ovarian cancer is recognized as a paradigm for tumor-associated immune suppression" [75] and the CGFI points 3. and 5. above and 6 . below are paths that may well reverse this aspect of tumor promotion, providing both "immune-activating strategies [and] elimination of immune-suppressing mechanisms" for better immunotherapy of ovarian cancer [76].

\section{Treat micrometastasis-seeded peritoneum surface with imiquimod lavage:}

Primary EOC surgery strives to remove as much tumor as possible but the risk/benefit for best extent of blind radicality of resection is still uncertain [77]. Often unseen [not visible to the naked eye] micrometastases are left, seeded throughout the peritoneum. These go on to kill. To combat these micrometastases the current practice of open perioperative hyperthermic peritoneal lavage with cytotoxic chemotherapy agents at the time of primary surgery has somewhat improved the prognosis of EOC and is generally well-tolerated $[78,79]$.

Imiquimod is a Toll-like receptor-7, TLR-7, agonist approved in USA/Canada and in the EU as a topical cream for treatment of basal cell carcinoma and other cutaneous malignancies $[80,81]$. It cannot be given systemically due to provocation of severe systemic inflammation. But it can be given topically without severe systemic effects, and this includes intraperitoneally in lavage fluid as a form of topical administration [82]. Since mice tolerated imiquimod peritoneal lavage without apparent discomfort [82] and topical imiquimod is effective in a wide variety of cancers resident in skin [81], it was suggested that open peritoneal lavage with an imiquimod solution be undertaken as treatment adjunct at the time of primary EOC surgery [83].

The data of Hackl et al. in 2011 [84] provides an interesting intersection with points 3 . and 5. above. They showed that treatment with TLR-7 agonists like imiquimod strongly suppressed Treg number and function [84].

\section{Block signaling at CXCR4/CXCL12 with plerixafor:}

As in many other cancers $[85,86]$, CXCR4 receptor signaling by 8 to $12 \mathrm{kDa}$ peptide chemokine CXCL12 [also termed stromal cellderived factor-1, SDF-1] is important to EOC growth, migration and angiogenesis [87-89]. "Abundant" CXCL12 protein was found in most EOC tissue samples but was absent in normal ovary tissue [87]. siRNA-mediated CXCL12 knockdown in an EOC cell line constitutively expressing CXCL12 and CXCR4 reduced proliferation in vitro, and tumor growth in vivo [88]. We can expect similar inhibition of human EOC by treatment with a low 
molecular weight, orally available CXCR4 antagonist, plerixafor. Plerixafor triggered necrosis and apoptosis in EOC cells [88]. "SDF1-CXCR4 interaction confers on EOC cells a remarkable potential to activate MMPs for subsequently invading the peritoneal cavity" [19], findings supported by immunohistochemical studies of others on the importance of CXCR4 in EOC [90]. This provides a happy intersection of captopril-based inhibition of MMP-2 and MMP-9 with plerixafor-based blocking of CXCR4 mediated stimulation of MMP-2 and MMP-9.

Since plerixafor is now approved and marketed for bone marrow stem cell mobilization prior to apharesis harvest for bone marrow transplantation, plerixafor was recently suggested as treatment adjunct in glioblastoma [86] where CXCR4 signaling also plays an important role. It is therefore now straightforward to suggest plerixafor in $\mathrm{EOC}$ based on the data above showing activity in a CXCL12 sensitive EOC rodent model and the ample evidence that the CXXCR4/CXCL12 axis plays an important role in growth stimulation in human EOC [87-89].

\section{Blocking bradykinin signaling with icatibant:}

Bradykinin is a $\sim 1 \mathrm{kDa}$, nine amino acid, inflammation-related signaling peptide, generated by proteolysis from a high molecular weight precursor kininogen [91]. Bradykinin is rendered inactive by $A C E$ leading to excess bradykinin during ACE inhibitor treatment. That causes the characteristic cough proclivity side effect of ACE inhibitors [91].

Ascites is common in EOC. Evidence points to the bradykinin system as at least contributory to this $[92,93]$. In humans, ascites development as a consequence of iatrogenic [nonmalignant] ovarian hyperstimulation occurs as a consequence of a peritoneal hyperpermeable state largely caused by excess ovary-produced bradykinin [94]. In experimental models, ovarian hyperstimulation ascites is, as expected, made worse by captopril [94]. Active bradykinin and inactive bradykinin catabolites are found in abundance in EOC ascites [95].

Icatibant is a bradykinin receptor blocker recently approved to treat acute hereditary angioedema bouts $[96,97]$. In addition icatibant is reportedly effective when used off-label to treat ACE inhibitor-related angioedema [98]. For both reasons identified above, a) the evidence that bradykinin plays at least a contributory role in development of EOC ascites, and b) the clear risk that we increase active bradykinin by the use of captopril [point 2. above], the use of a commercially marketed bradykinin inhibitor, icatibant, will be advisable.

\section{Conclusion}

To improve the prognosis in EOC an effort to comprehensively block growth factors that have been identified as active in human EOC as suggested in this paper may prove fruitful. Past research indicates that several already-approved and marketed drugs might do this. The nine drugs have no clearly discernable interaction with each other and none would be expected to interfere with concomitant current cytotoxic chemotherapy regimens although such cannot be excluded. Given the safety of the nine drugs individually, and the poor prognosis of an EOC seeded peritoneum, the risk of unexpected side effects or interaction I believe is worth taking.

\section{Aknowledgements}

Warm thanks to my colleagues for helpful discussions, kind support, and wise counsel.

\section{Abbreviations}

ACE: angiotensin converting enzyme; CGFI: Comprehensive Growth Factor Inhibition

EOC: epithelial ovarian cancer; interleukin-2, IL-2; TLR-7, Toll-like receptor-7.

\section{Article History}

Received: 21-Dec-2011 Accepted: 23-Jan-2012

Published: 14-Feb-2012

\section{References}

1. Bookman, M. A. Developmental chemotherapy and management of recurrent ovarian cancer. J Clin Oncol 21, 149s-167s.

2. Guppy, A. E. et al. Epithelial ovarian cancer: a review of current management. Clin Oncol (R Coll Radiol) 17, 399-411.

3. Chua, T. C. et al. Intraoperative hyperthermic intraperitoneal chemotherapy after cytoreductive surgery in ovarian cancer peritoneal carcinomatosis: systematic review of current results. J Cancer Res Clin Oncol 135, 1637-1645.

4. Mantia-Smaldone, G. M. et al. Targeted treatment of recurrent platinum-resistant ovarian cancer: current and emerging therapies. Cancer Manag Res 3, 25-38.

5. Stern, Capt JCS. Civil Military Operations \& Military Information Support Operations Coordination: A Non-Kinetic Ballast for Disciplined Counterinsurgency Operations. smallwarsjournal.com 2011.

6. Kast RE. Endothelin-1 inhibition by ambrisentan as a potential treatment adjunct after debulking surgery in epithelial ovarian cancer. Oncol Res. 17, 383-6.

7. Rosanò $L$, et al. Acquisition of chemoresistance and EMT phenotype is linked with activation of the endothelin A receptor pathway in ovarian carcinoma cells. Clin Cancer Res. 2011 Apr 17, 2350-60.

8. Bagnato, A. et al. Epithelial-mesenchymal transition in ovarian cancer progression: a crucial role for the endothelin axis. Cells Tissues Organs 185, 85-94.

9. Frampton, J. E. Ambrisentan. Am J Cardiovasc Drugs 11 215-226.

10. Ahmed, N. et al. Overexpression of alpha(v)beta6 integrin in serous epithelial ovarian cancer regulates extracellular matrix degradation via the plasminogen activation cascade. Carcinogenesis 23, 237-244.

11. Murthi $P$, et al. Plasminogen fragmentation and increased production of extracellularmatrixdegrading proteinases are associated with serous epithelial ovarian cancer progression. Gynecol Oncol. 92, 80-8.

12. Choi $\mathrm{JH}$, et al. Gonadotropins activate proteolysis and increase invasion through protein kinase $A$ and phosphatidylinositol 3-kinase pathways in human epithelial ovarian cancer cells. Cancer Res. 66, 3912-20.

13. Cowden Dahl KD, et al. Matrix metalloproteinase 9 is a mediator of epidermal growth factor-dependent e-cadherin loss in ovarian carcinoma cells. Cancer Res. 68,4606-13

14. Kwon, Y. et al. Differential expressions of adhesive molecules and proteases define mechanisms of ovarian tumor cell matrix penetration/invasion. PLOS One 6, e18872.

15. Alshenawy, H. A. Immunohistochemical expression of epidermal growth factor receptor, E-cadherin, and matrix metalloproteinase-9 in ovarian epithelial cancer and relation to patient deaths. Ann Diagn Pathol 14, 387-395. 
16. Wang, F. Q. et al. Vascular endothelial growth factorregulated ovarian cancer invasion and migration involves expression and activation of matrix metalloproteinases. Int J Cancer 118, 879-888.

17. Demeter, A. et al. Molecular prognostic markers in recurrent and in non-recurrent epithelial ovarian cancer. Anticancer Res 25, 2885-2889.

18. Kamat, A. A. et al. The clinical relevance of stromal matrix metalloproteinase expression in ovarian cancer. Clin Cancer Res 12, 1707-1714.

19. Yuecheng, Y. et al. Stromal-cell derived factor-1 regulates epithelial ovarian cancer cell invasion by activating matrix metalloproteinase- 9 and matrix metalloproteinase-2. Eur J Cancer Prev 16, 430-435.

20. Sillanpaa, S. et al. Prognostic significance of matrix metalloproteinase-9 (MMP-9) in epithelial ovarian cancer. Gynecol Oncol 104, 296-303.

21. Sakata $\mathrm{K}$, et al. Expression of matrix metalloproteinases (MMP-2, MMP-9, MT1-MMP) and their inhibitors (TIMP-1, TIMP-2) in common epithelial tumors of the ovary. Int J Oncol. 17, 673-81.

22. Hudson, L. G. et al. EGF-receptor regulation of matrix metalloproteinases in epithelial ovarian carcinoma. Future Oncol 5, 323-338.

23. Zhao $Y$, et al. RhoC expression level is correlated with the clinicopathological characteristics of ovarian cance and the expression levels of ROCK-I, VEGF, and MMP9. Gynecol Oncol. 116, 563-71.

24. Nakada, M. et al. The role of matrix metalloproteinases in glioma invasion. Front Biosci 8, e261-269.

25. Sorbi, D. et al. Captopril inhibits the 72 kDa and 92 kDa matrix metalloproteinases. Kidney Int 44, 1266-1272.

26. Young JB. Angiotensin-converting enzyme inhibitors in heart failure: newstrategies justified by recent clinical trials. Int J Cardiol. 43, 151-63.

27. Rooprai, H. K. et al. Evaluation of the effects of swainsonine, captopril, tangeretin and nobiletin on the biological behaviour of brain tumour cells in vitro. Neuropathol Appl Neurobiol 27, 29-39.

28. Nakagawa, T. et al. Captopril inhibits glioma cell invasion in vitro: involvement of matrix metalloproteinases. Anticancer Res 15, 1985-1989.

29. Geppetti, P. et al. Acute oral captopril inhibits angiotensin converting enzyme activity in human cerebrospinal fluid. J Hypertens 5, 151-154.

30. Inoue, N. et al. Effect of angiotensin-converting enzyme inhibitor on matrix metalloproteinase-9 activity in patients with Kawasaki disease. Clin Chim Acta 411 , 267-269.

31. Yamamoto D, et al. Inhibitory profiles of captopril on matrix metalloproteinase-9 activity. Eur J Pharmacol. 588, 277-9. PubMed PMID: 18501888

32. Reinhardt, D. et al. Cardiac remodelling in end stage heart failure: upregulation of matrix metalloproteinase (MMP) irrespective of the underlying disease, and evidence for a direct inhibitory effect of ACE inhibitors on MMP. Heart 88, 525-530.

33. Kichuk MR, et al. Angiotensin-converting nzyme inhibitors promote nitric oxide production in coronary microvessels from failing explanted human hearts. $A m$ Cardiol. 80, 137A-142A. PubMed PMID: 9293967.

34. Efsen, E. et al. Ramiprilate inhibits functional matrix metalloproteinase activity in Crohn's disease fistulas. Basic Clin Pharmacol Toxicol 109, 208-216.

35. Tang, Q. et al. CD4+Foxp3+ regulatory $\mathbf{T}$ cell therapy in transplantation. J Mol Cell Biol 4, 11-21.

36. Knutson $\mathrm{KL}$, et al. Immunologic principles and immunotherapeutic approaches in ovarian cancer. Hematol Oncol Clin North Am. 17, 1051-73

37. Curiel $\mathrm{TJ}$, et al. Specific recruitment of regulatory $\mathbf{T}$ cells in ovarian carcinoma fosters immune privilege and predicts reduced survival. Nat Med. 10, 942-9

38. Preston, C. C. et al. Immunity and immune suppression in human ovarian cancer. Immunotherapy 3, 539-556.

39. Gavalas NG, et al. Immune response in ovarian cancer: how is the immune system involved in prognosis and therapy: potential for treatment utilization. Clin Dev Immunol. 2010, 791603.

40. Barnett, B. et al. Regulatory T cells in ovarian cancer: biology and therapeutic potential. Am J Reprod Immunol 54, 369-377.
41. Wolf $\mathrm{D}$, et al. The expression of the regulatory $\mathbf{T}$ cellspecific forkhead box transcription factor FoxP3 is associated with poor prognosis in ovarian cancer. Clin Cancer Res. 11, 8326-31.

42. Sato, E. et al. Intraepithelial CD8+ tumor-infiltrating lymphocytes and a high CD8+/regulatory $\mathrm{T}$ cell ratio are associated with favorable prognosis in ovarian cancer. Proc Natl Acad Sci U S A 102, 18538-18543.

43. Kryczek I, et al. Relationship between B7-H4, regulatory $T$ cells, and patient outcome in human ovarian carcinoma. Cancer Res. 67, 8900-5.

44. Leveque $L$, et al. Interleukin 2-mediated conversion of ovarian cancer-associated CD4+ regulatory $\mathrm{T}$ cells into proinflammatory interleukin 17-producing helper $\mathbf{T}$ cells. J Immunother. 32, 101-8.

45. Sehouli, J. et al. Epigenetic quantification of tumorinfiltrating T-lymphocytes. Epigenetics 6, 236-246.

46. Söderlund J, et al. Acyclovir inhibition of IDO to decrease Tregs as a glioblastoma treatment adjunct. $J$

Neuroinflammation. 7, 44. PubMed PMID: 20691089; PubMed Central PMCID: PMC2925358.

47. Cannon $\mathrm{MJ}$, et al. Dendritic cell vaccination against ovarian cancer--tipping the Treg/TH17 balance to therapeutic advantage? Expert Opin Biol Ther. 11, 441-5. Munn, D. H. et al. Indoleamine 2,3-dioxygenase and tumor-induced tolerance. J Clin Invest 117, 1147-1154.

49. Kast, R. E. et al. Indoleamine 2,3-dioxygenaseexpressing mature human monocyte-derived dendritic cells expand potent autologous regulatory $\mathbf{T}$ cells: consideration of triamterene to treat lupus. Med Hypotheses 74, 957

50. Müller AC, et al. Mechanisms by which acyclovir reduces the oxidative neurotoxicity and biosynthesis of quinolinic acid. Life Sci. 80, 918-25.

51. Sperner-Unterweger B, et al. Enhanced tryptophan degradation in patients with ovarian carcinoma correlates with several serum soluble immune activation markers. Immunobiology. 216 296-301.

52. Schroecksnadel, K. et al. Tryptophan degradation in patients with gynecological cancer correlates with immune activation. Cancer Lett 223, 323-329.

53. Wicherek, L. et al. Analysis of Treg cell population alterations in the peripheral blood of patients treated surgically for ovarian cancer - a preliminary report. Am J Reprod Immunol 66, 444-450.

54. Wu, X. et al. The immunologic aspects in advanced ovarian cancer patients treated with paclitaxel and carboplatin chemotherapy. Cancer Immunol Immunother 59, 279-291.

55. Reinartz $S$, et al. Suppressive activity rather than frequency of FoxP3 $(+)$ regulatory $T$ cells is essential for CA-125-specific T-cell activation after abagovomab treatment. Hum Immunol. 71, 36-44.

56. Vermeij $\mathrm{R}$, et al. Tumor-infiltrating cytotoxic $\mathbf{T}$ lymphocytes as independent prognostic factor in epithelial ovarian cancer with wilms tumor protein 1 overexpression. J Immunother. 34, 516-23.

57. Barnett, B. et al. Regulatory $\mathbf{T}$ cells in ovarian cancer: biology and therapeutic potential. Am J Reprod Immunol 54, 369-377

58. Suh JJ, Pettinati HM, Kampman KM, O'Brien CP. The status of disulfiram: a half of a century later. J Clin

Psychopharmacol. 26, 290-302.

59. Kast RE, Belda-Iniesta C. Suppressing glioblastoma stem cell function by aldehyde dehydrogenase inhibition with chloramphenicol or disulfiram as a new treatment adjunct: an hypothesis. Curr Stem Cell Res Ther. 4, 314-7.

60. Ma, I. et al. The role of human aldehyde dehydrogenase in normal and cancer stem cells. Stem Cell Rev 7, 292-306

61. Moreb JS, et al. The enzymatic activity of human aldehyde dehydrogenases $1 A 2$ and 2 (ALDH1A2 and ALDH2) is detected by Aldefluor, inhibited by diethylaminobenzaldehyde and has significant effects on cell proliferation and drug resistance. Chem Biol Interact. [Epub ahead of print] PMID: 22079344

62. Deng $S$, et al. Distinct expression levels and patterns of stem cell marker, aldehyde dehydrogenase isoform 1 (ALDH1), in human epithelial cancers. PLOS One. 5 e10277. PubMed PMID: 20422001; PubMed Central PMCID: PMC2858084. 
63. Kryczek, I. et al. Expression of aldehyde dehydrogenase and CD133 defines ovarian cancer stem cells. Int J Cancer 130, 29-39.

64. Landen, C. N., Jr. et al. Targeting aldehyde dehydrogenase cancer stem cells in ovarian cancer. Mol Cancer Ther 9, 3186-3199.

65. Silva IA, et al. Aldehyde dehydrogenase in combination with CD133 defines angiogenic ovarian cancer stem cells that portend poor patient survival. Cancer Res. 71, 3991-4001. PubMed PMID: 21498635; PubMed Central PMCID: PMC3107359.

66. Rizzo, S. et al. Ovarian cancer stem cell-like side populations are enriched following chemotherapy and overexpress EZH2. Mol Cancer Ther 10, 325-335.

67. Miller, S. C. et al. The role of melatonin in immunoenhancement: potential application in cancer. Int J Exp Pathol 87, 81-87.

68. Maestroni, G. J. The immunotherapeutic potential of melatonin. Expert Opin Investig Drugs 10, 467-476.

69. Srinivasan, V. et al. Melatonin, immune function and cancer. Recent Pat Endocr Metab Immune Drug Discov 5 , 109-123.

70. Cardinali, D. P. et al. Melatonin and its analogs in insomnia and depression. J Pineal Res

71. Kast RE, Altschuler EL. Co-administration of ramelteon and fluvoxamine to increase levels of interleukin-2. Med Hypotheses. 2006;67(6):1389-90.

72. Carrillo-Vico, A. et al. Melatonin counteracts the inhibitory effect of PGE2 on IL-2 production in human lymphocytes via its $\mathbf{m t} \mathbf{1}$ membrane receptor. FASEB J 17, 755-757.

73. García-Mauriño S, et al. Correlation between nuclear melatonin receptor expression and enhanced cytokine production in human lymphocytic and monocytic cell lines. J Pineal Res. 29, 129-37.

74. Wei, S. et al. Interleukin-2 administration alters the CD4+FOXP3+ T-cell pool and tumor trafficking in patients with ovarian carcinoma. Cancer Res 67, 7487-7494.

75. Cannon, M. J. et al. Cellular immunotherapy for ovarian cancer. Expert Opin Biol Ther 9, 677-688.

76. Yigit, R. et al. Ovarian cancer creates a suppressive microenvironment to escape immune elimination. Gynecol Oncol 117, 366-372.

77. Elattar, A. et al. Optimal primary surgical treatment for advanced epithelial ovarian cancer. Cochrane Database Syst Rev, CD007565.

78. Chua TC, et al. Intraoperative hyperthermic intraperitoneal chemotherapy after cytoreductive surgery in ovarian cancer peritoneal carcinomatosis: systematic review of urrent results. J Cancer Res Clin Oncol. 135, 1637-45.

79. Sugarbaker $\mathrm{PH}$. Comprehensive management of peritoneal surface malignancy using cytoreductive surgery and perioperative intraperitoneal chemotherapy: the Washington Cancer Institute approach. Expert Opin Pharmacother. 10, 1965-77. PubMed PMID: 19563273.

80. David, C. V. et al. Imiquimod: a review of off-label clinical applications. J Drugs Dermatol 10, 1300-1306.

81. Love, W. E. et al. Topical imiquimod or fluorouracil therapy for basal and squamous cell carcinoma: a systematic review. Arch Dermatol 145, 1431-1438.

82. Altintas, D. et al. Efficacy of imiquimod, an immunomodulatory agent, on experimental endometriosis. Fertil Steril 90, 401-405.

83. Kast, R. E. et al. Intraperitoneal lavage with imiquimod to eliminate epithelial ovarian cancer micro metastases. Med Hypotheses 70, 1223-1224.

84. Hackl, D. et al. Activation of dendritic cells via TLR7 reduces Foxp3 expression and suppressive function in induced Tregs. Eur J Immunol 41, 1334-1343.

85. Teicher BA, Fricker SP. CXCL12 (SDF-1)/CXCR4 pathway in cancer. Clin Cancer Res. 16, 2927-31.

86. Kast RE. Profound blockage of CXCR4 signaling at multiple points using the synergy between plerixafor mirtazapine, and clotrimazole as a new glioblastoma treatment adjunct. Turk Neurosurg. 20, 425-9.

87. Scotton, C. J. et al. Multiple actions of the chemokine CXCL12 on epithelial tumor cells in human ovarian cancer. Cancer Res 62, 5930-5938.

88. Righi $\mathrm{E}$, et al. CXCL12/CXCR4 blockade induces multimodal antitumor effects that prolong survival in an immunocompetent mouse model of ovarian cancer. Cancer Res. 71, 5522-34.

89. Jiang, Y. P. et al. Role of CXCL12 in metastasis of human ovarian cancer. Chin Med J (Engl) 120, 1251-1255.

90. Kulbe, H. et al. A Dynamic Inflammatory Cytokine Network in the Human Ovarian Cancer

Microenvironment. Cancer Res 72, 66-75.

91. Bryant, J. W. et al. Human plasma kallikrein-kinin system: physiological and biochemical parameters. Cardiovasc Hematol Agents Med Chem 7, 234-250.

92. Wunderer G, et al. Human Ile-Ser-bradykinin, identical with rat T-kinin, is a major permeability factor in ovarian carcinoma ascites. Biol Chem Hoppe Seyler. 367, 1231-4. PubMed PMID: 3828072.

93. Wunderer, G. et al. T-kinin in human ovarian carcinoma ascites. Adv Exp Med Biol 247B, 109-114.

94. Kobayashi $\mathrm{H}$, et al. The kallikrein-kinin system, but not vascular endothelial growth factor, plays a role in the increased vascular permeability associated with ovarian hyperstimulation syndrome. J Mol Endocrinol. 20, 363-74.

95. Matsumura, Y. et al. Degradation pathway of kinins in tumor ascites and inhibition by kininase inhibitors: analysis by HPLC. Agents Actions 29, 172-180.

96. Deeks, E. D. Icatibant. Drugs 70, 73-81.

97. Gras, J. Icatibant for hereditary angioedema. Drugs Today (Barc) 45, 855-864.

98. Schmidt, P. W. et al. Treatment of angiotensinconverting enzyme inhibitor-related angioedema with the bradykinin B2 receptor antagonist icatibant. J Am Acad Dermatol 63, 913-914. 